\title{
ANALYTICAL VALIDATION OF THE FINITE ELEMENT METHOD MODEL FOR LAPLACE EQUATION
}

\author{
Ewa Wegrzyn-Skrzypczak ${ }^{1}$, Tomasz Skrzypczak ${ }^{2}$ \\ ${ }^{I}$ Institute of Mathematics, Czestochowa University of Technology \\ Czestochowa, Poland \\ ${ }^{2}$ Institute of Mechanics and Machine Design Fundamentals, Czestochowa University of Technology \\ Czestochowa, Poland \\ ewa.skrzypczak@im.pcz.pl,t.skrzypczak@imipkm.pcz.pl
}

Received: 8 September 2018; Accepted: 1 October 2019

\begin{abstract}
The presented paper is focused on the comparison of the numerical solution of the Laplace equation in a two-dimensional space with the results obtained with the use of the analytical method. The results of the numerical model are computed on the base of the Finite Element Method. The analytical solution of the considered equation is obtained using the Fourier series. Finally the results of both methods are compared in order to verify the accuracy of numerical implementation.
\end{abstract}

MSC 2010: 35J25, 35J47, 65M60

Keywords: Laplace equation, Fourier's method, Finite Element Method

\section{Introduction}

Mathematical models of many physical phenomena (in fields such as electricity mechanics, magnetism, thermodynamics, etc.) are described using second order linear partial differential equations, which are called equations of mathematical physics. The task of determining functions that describe these equations leads to an infinite number of solutions. For specific cases, special solutions that meet certain initial and boundary conditions are sought [1].

In order to solve equations of mathematical physics, the analytical or numerical methods are used. Analytical methods allow one to obtain an exact solution of the considered problem, but they cannot be used in all cases. Numerical methods can be used in almost every case, but they give approximated results.

One of the most popular analytical methods used to solve equations of mathematical physics is the Fourier method. It is based on finding a solution in the form of the product of functions where each of them is dependent only on one variable. The use of appropriate boundary conditions makes it possible to determine the value of constants appearing in the solutions $[2,3]$. 
One of the numerical methods widely used in the solving of equations of mathematical physics is the Finite Element Method (FEM). In this method, the considered area is divided into small and simple-shaped sub-areas, called finite elements, in which approximation functions are determined. These functions are algebraic polynomials, which are determined on the basis of the rules of approximation interpolation. The form of the approximation functions depends on the number and positions of the nodes located within the finite element. Unknown variables in the nodes, such as temperature values are determined with the use of known boundary conditions. Finally the discrete set of the nodal variables is calculated as the solution of the system of algebraic equations [4-6].

Many authors from different fields compare results of their numerical models with analytical solutions or with the numerical solutions obtained on the basis of different models [7]. The comparison with the analytical solution proves the mathematical accuracy of developed model while comparing obtained results with the numerical solution of considered problem proves the accuracy of implementation of used numerical method. To prove the accuracy of a used numerical model in the simulation of the behaviour of real systems or objects, the comparison with an experiment is often performed [8].

In order to verify the correctness of presented results obtained with the use of FEM, they are compared with the analytical solution of the considered equation. A similar comparison, but for a one-dimensional case, was done in [9]. An FEM solution is obtained with the use of an originally developed computer program.

\section{Formulation of the problem}

Let's consider a two-dimensional area $0<x<a, 0<y<b$ in rectangular coordinate systems. At the boundaries of the region the Dirichlet boundary conditions are assigned [10]:

$$
\begin{gathered}
u(0, y)=u(a, y)=0 \\
u(x, 0)=f(x) \\
u(x, b)=g(x)
\end{gathered}
$$

The distribution of the sought function $u(x, y)$ within the considered area is described by the Laplace equation in the form:

$$
\frac{\partial^{2} u}{\partial x^{2}}+\frac{\partial^{2} u}{\partial y^{2}}=0
$$


On the basis of conditions (1)-(3), it was assumed, that $f(0)=g(0)=0$ and $f(a)=g(a)=0$. The scheme of the considered problem is presented in Figure 1 .

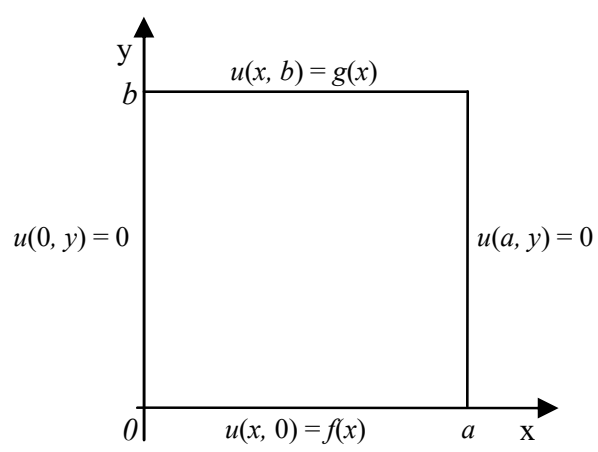

Fig. 1. Scheme of the problem

\section{Analytical solution}

In order to find the analytical solution of Laplace equation (4) with the boundary conditions (1)-(3) the Fourier method is used. The solution of the equation (4) has a form of the following function $[1,2,10]$ :

$$
u(x, y)=\sum_{k} X_{k}(x) Y_{k}(y)
$$

On the assumption that each component of the sum (5) meets the differential equation (4), the following relation is obtained:

$$
X_{k}{ }^{\prime \prime}(x) Y_{k}(y)+X_{k}(x) Y_{k}{ }^{\prime \prime}(y)=0
$$

then

$$
\frac{X_{k}{ }^{\prime \prime}(x)}{X_{k}(x)}=-\frac{Y_{k}{ }^{\prime \prime}(y)}{Y_{k}(y)}=\gamma_{k}
$$

where $\gamma_{k}$ is constant. Moreover, each component of the sum (5) must satisfy the condition (1), therefore:

$$
\begin{aligned}
& X_{k}(0) Y_{k}(y)=0 \\
& X_{k}(a) Y_{k}(y)=0
\end{aligned}
$$

This means that the functions $X_{k}$ are the solution of the following problem: 


$$
\left\{\begin{array}{c}
X_{k}{ }^{\prime \prime}(x)-\gamma_{k} X_{k}(x)=0 \\
X_{k}(0)=X_{k}(a)=0
\end{array}\right.
$$

On the assumption that $\gamma_{k}<0$ (for $\gamma_{k} \geq 0$ the trivial solutions of the equation are obtained) finally it follows that:

$$
\gamma_{k}=-\left(\frac{k \pi}{a}\right)^{2}, k \in Z
$$

and the solutions of equation (10) are functions $X_{k}$ that takes the form (11):

$$
X_{k}(x)=\sin \frac{k \pi}{a} x
$$

By substituting (11) and (12) into equation (7) and on the assumption that $Y_{k}(y)=e^{\alpha_{k} y}$, where $\alpha_{k}= \pm \frac{k \pi}{a}$, functions $Y_{k}$ are obtained:

$$
Y_{k}(y)=c_{k} e^{\frac{k \pi}{a} y}+d_{k} e^{\frac{-k \pi}{a} y}
$$

Finally, the solution of equation (6) takes the form [10]:

$$
u(x, y)=\sum_{k=1}^{\infty} \sin \frac{k \pi}{a} x\left(c_{k} e^{\frac{k \pi}{a} y}+d_{k} e^{\frac{-k \pi}{a} y}\right)
$$

In order to determine the values of constants $c_{k}$ and $d_{k}$ boundary conditions (2), (3) are used. Therefore:

$$
\begin{gathered}
c_{k}=-\frac{\int_{0}^{a}\left[f(x) e^{-\frac{k \pi b}{a}}-g(x)\right] \sin \frac{k \pi}{a} x d x}{a \sinh \frac{k \pi b}{a}} \\
d_{k}=-\frac{\int_{0}^{a}\left[g(x)-f(x) e^{\frac{k \pi b}{a}}\right] \sin \frac{k \pi}{a} x d x}{a \sinh \frac{k \pi b}{a}}
\end{gathered}
$$

for $k=1,2, \ldots$. 


\section{Numerical solution}

To find the numerical solution of the equation (4) with conditions (1)-(3), the finite element method is used.

Equation (4) is multiplied by the function $w(x, y)$ and then integrated over domain $\Omega$ [4-6]:

$$
\int_{\Omega} w\left(\frac{\partial^{2} u}{\partial x^{2}}+\frac{\partial^{2} u}{\partial y^{2}}\right) d \Omega=0
$$

Within the equation (17) are the terms of the second order. To lower the order of the equation it is necessary to use Green's theorem as follows:

$$
\int_{\Omega} w\left(\frac{\partial^{2} u}{\partial x^{2}}+\frac{\partial^{2} u}{\partial y^{2}}\right) d \Omega=-\int_{\Omega}\left(\frac{\partial w}{\partial x}\left(\frac{\partial u}{\partial x}\right)+\frac{\partial w}{\partial y}\left(\frac{\partial u}{\partial y}\right)\right) d \Omega+\int_{\Gamma} w\left(\frac{\partial u}{\partial x} n_{x}+\frac{\partial u}{\partial y} n_{y}\right) d \Gamma
$$

where $n_{x}, n_{y}$ are components of the vector normal to the external boundary.

In the case of the integral function, which appears in the second part on the right side of the equation (18), the substitution $\frac{\partial u}{\partial x} n_{x}+\frac{\partial u}{\partial y} n_{y}=-q$ can be made in order to write the following relation:

$$
\int_{\Omega} w\left(\frac{\partial^{2} u}{\partial x^{2}}+\frac{\partial^{2} u}{\partial y^{2}}\right) d \Omega=-\int_{\Omega}\left(\frac{\partial w}{\partial x}\left(\frac{\partial u}{\partial x}\right)+\frac{\partial w}{\partial y}\left(\frac{\partial u}{\partial y}\right)\right) d \Omega-\int_{\Gamma} w q d \Gamma
$$

After substitution of (19) to equation (17) it can be written:

$$
\int_{\Omega}\left(\frac{\partial w}{\partial x}\left(\frac{\partial u}{\partial x}\right)+\frac{\partial w}{\partial y}\left(\frac{\partial u}{\partial y}\right)\right) d \Omega=-\int_{\Gamma} w q d \Gamma
$$

In order to perform spatial discretization, domain $\Omega$ is spatially discretized with the use of triangular finite elements. The nodes, where the values of the function $u$ are determined, are located in the vertices of each finite element. In order to simplify the mathematical operations, each finite element is transformed from the real space $x, y$ to the normalized one $\xi, \eta$. Linear shape functions in a normalized triangle $N_{k}, k=1, \ldots, 3$, take the form [4-6]:

$$
\tilde{N}_{1}(\xi, \eta)=1-\xi-\eta, \quad \tilde{N}_{2}(\xi, \eta)=\xi, \quad \tilde{N}_{3}(\xi, \eta)=\eta
$$


The derivatives of the shape function (21) with respect to $\xi, \eta$ are used to determine the Jacobian, which is defined as follows:

$$
\mathbf{J}=\left[\begin{array}{ll}
\frac{\partial x}{\partial \xi} & \frac{\partial x}{\partial \eta} \\
\frac{\partial y}{\partial \xi} & \frac{\partial y}{\partial \eta}
\end{array}\right]=\left[\begin{array}{cc}
\frac{\partial \tilde{N}_{i}}{\partial \xi} x_{i} & \frac{\partial \tilde{N}_{i}}{\partial \eta} x_{i} \\
\frac{\partial \tilde{N}_{i}}{\partial \xi} y_{i} & \frac{\partial \tilde{N}_{i}}{\partial \eta} y_{i}
\end{array}\right], \quad i=1 \ldots 3
$$

In order to determine derivatives of the shape functions with respect to variables $x, y$, the following relation must be used:

$$
\left[\begin{array}{ccc}
\frac{\partial N_{1}}{\partial x} & \frac{\partial N_{2}}{\partial x} & \frac{\partial N_{3}}{\partial x} \\
\frac{\partial N_{1}}{\partial y} & \frac{\partial N_{2}}{\partial y} & \frac{\partial N_{3}}{\partial y}
\end{array}\right]=\left(\mathbf{J}^{-1}\right)^{T}\left[\begin{array}{ccc}
\frac{\partial N_{1}}{\partial \xi} & \frac{\partial N_{2}}{\partial \xi} & \frac{\partial N_{3}}{\partial \xi} \\
\frac{\partial N_{1}}{\partial \eta} & \frac{\partial N_{2}}{\partial \eta} & \frac{\partial N_{3}}{\partial \eta}
\end{array}\right]
$$

where

$$
\mathbf{J}^{-1}=\frac{1}{\operatorname{det} \mathbf{J}}\left[\begin{array}{cc}
J_{1,1} & -J_{0,1} \\
-J_{1,0} & J_{0,0}
\end{array}\right]
$$

Using the Galerkin formulation test functions $w(x, y)$ can be written as:

$$
w_{i}(x, y)=N_{i}(x, y) \text { for } i=1, \ldots, 3
$$

the equation (20) takes a form [4-6]:

$$
\int_{\Omega^{(e)}}\left(\frac{\partial N_{i}}{\partial x} \frac{\partial N_{j}}{\partial x}+\frac{\partial N_{i}}{\partial y} \frac{\partial N_{j}}{\partial y}\right) d \Omega^{(e)} u=-\int_{\Gamma^{(e)}} N_{i} q d \Gamma^{(e)}
$$

where $(e)$ is a triangular finite element.

The integrals from the equation (26) can be written in the matrix form:

$$
\mathbf{K}^{(e)}=\int_{\Omega^{(e)}}\left[\begin{array}{llll}
\frac{\partial N_{1}}{\partial x} \frac{\partial N_{1}}{\partial x}+\frac{\partial N_{1}}{\partial y} \frac{\partial N_{1}}{\partial y} & \frac{\partial N_{1}}{\partial x} \frac{\partial N_{2}}{\partial x}+\frac{\partial N_{1}}{\partial y} \frac{\partial N_{2}}{\partial y} & \frac{\partial N_{1}}{\partial x} \frac{\partial N_{3}}{\partial x}+\frac{\partial N_{1}}{\partial y} \frac{\partial N_{3}}{\partial y} \\
\frac{\partial N_{2}}{\partial x} \frac{\partial N_{1}}{\partial x}+\frac{\partial N_{2}}{\partial y} \frac{\partial N_{1}}{\partial y} & \frac{\partial N_{2}}{\partial x} \frac{\partial N_{2}}{\partial x}+\frac{\partial N_{2}}{\partial y} \frac{\partial N_{2}}{\partial y} & \frac{\partial N_{2}}{\partial x} \frac{\partial N_{3}}{\partial x}+\frac{\partial N_{2}}{\partial y} \frac{\partial N_{3}}{\partial y} \\
\frac{\partial N_{3}}{\partial x} \frac{\partial N_{1}}{\partial x}+\frac{\partial N_{3}}{\partial y} \frac{\partial N_{1}}{\partial y} & \frac{\partial N_{3}}{\partial x} \frac{\partial N_{2}}{\partial x}+\frac{\partial N_{3}}{\partial y} \frac{\partial N_{2}}{\partial y} & \frac{\partial N_{3}}{\partial x} \frac{\partial N_{3}}{\partial x}+\frac{\partial N_{3}}{\partial y} \frac{\partial N_{3}}{\partial y}
\end{array}\right] d \Omega^{(e)}
$$




$$
\mathbf{B}^{(e)}=-\int_{\Gamma^{(e)}}\left[\begin{array}{c}
N_{1} \\
N_{2} \\
N_{3}
\end{array}\right] q d \Gamma^{(e)}
$$

where $\mathbf{K}^{(e)}$ is the coefficient matrix of the element and $\mathbf{B}^{(e)}$ is the right hand side vector of the element.

Finally, one can write the following local matrix equation:

$$
\mathbf{K}^{(e)} \mathbf{U}^{(e)}=\mathbf{B}^{(e)}
$$

The aggregation procedure of the local equations allows one to obtain the following global system of equations:

$$
\mathbf{K U}=\mathbf{B}
$$

The equation (30) requires modification of calculations at every step by introducing the boundary conditions.

\section{Examples of calculation}

In order to verify the accuracy of numerical implementation, the results of the solution of equation (4) with the boundary conditions (1)-(3) obtained with the use of analytical and numerical methods are compared. In the case of an analytical solution obtained using the Fourier method, the calculations were carried out using formulas (14)-(16). To obtain a numerical solution, the finite element method described in chapter 4 was used.

The calculations were carried out for a square-shaped area with a side length of $1 \mathrm{~m}$ with the boundary conditions shown in Figure 2.

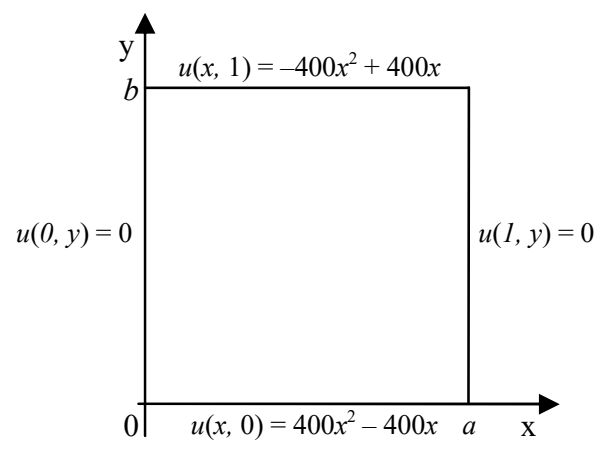

Fig. 2. Boundary conditions

The calculations were made with the use of three meshes of different qualities. In the first case, the average dimension of the finite element was $h_{e}=0.1 \mathrm{~m}$ and 
the mesh contained 142 nodes, in the second case $h_{e}=0.01 \mathrm{~m}$ and 13459 nodes, in the last one $h_{e}=0.001 \mathrm{~m}$ and 1323240 nodes.

The results of the analytical and numerical solutions obtained for the second case are shown in Figures 3 and 4.

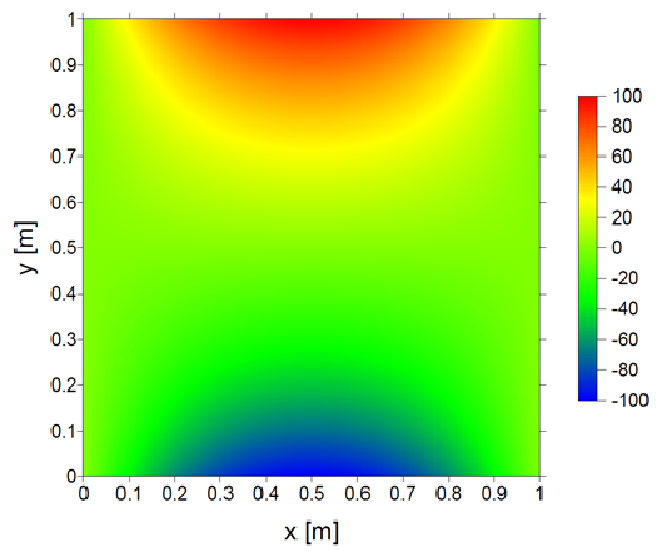

Fig. 3. Distribution of function $u(x, y)$ obtained using the Fourier series (the second case)

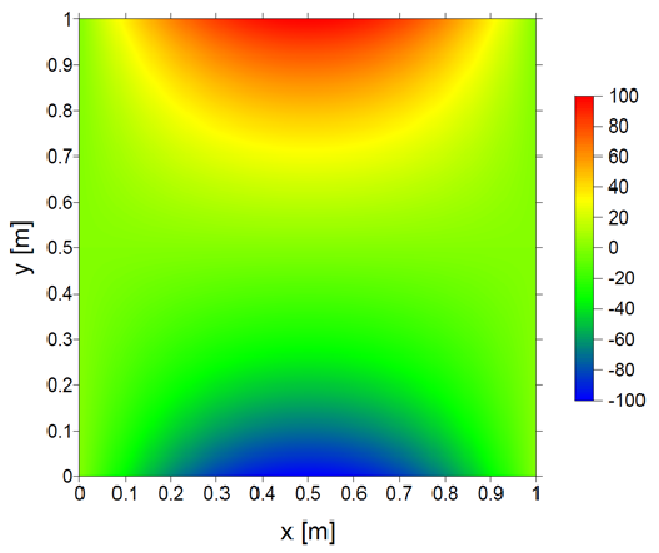

Fig. 4. Distribution of function $u(x, y)$ obtained using the FEM (the second case)

In order to compare the results of analytical and numerical calculations, the percentage differences between the results obtained for the three cases were calculated using the following relationship:

$$
u_{\text {error }}=100 \frac{\left|u_{F E M}(x, y)-u_{\text {Analytical }}(x, y)\right|}{\left(u_{\max }-u_{\text {min }}\right)_{\text {Analytical }}}
$$

They are shown in Figures 5-7. 


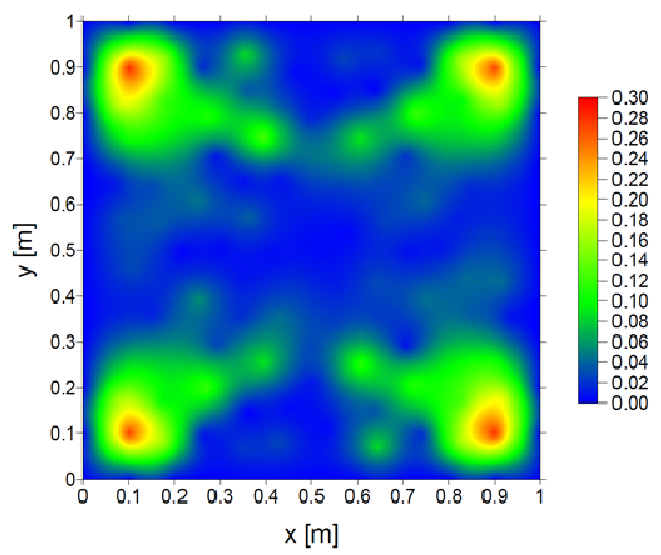

Fig. 5. Distribution of percentage differences of $u(x, y)$ for the first case

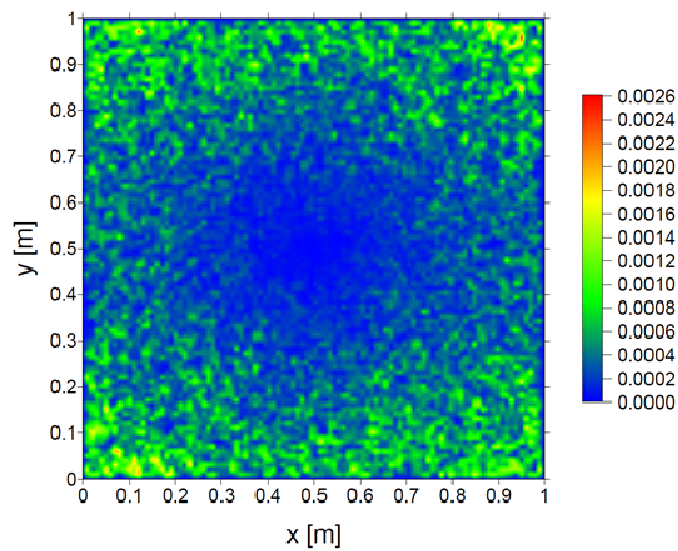

Fig. 6. Distribution of percentage differences for $u(x, y)$ for the second case

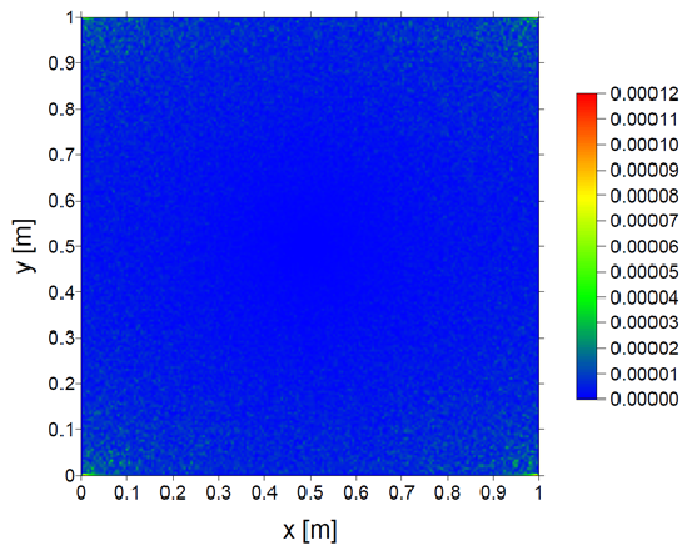

Fig. 7. Distribution of percentage differences of $u(x, y)$ for the third case 


\section{Conclusions}

The results of calculation obtained for the analytical (Fig. 3) and numerical (Fig. 4) solutions are very similar. In the first variant, the maximum deviations between solutions reach $0.3 \%$, in the second variant $0.0026 \%$, and in the last one $0.00012 \%$. This confirms the correctness of both models and shows the significant influence of the mesh quality on the accuracy of the FEM model.

The presented model can be modified and used to obtain solutions for threedimensional problems. In the case of the analytical model, the only limitation is the complexity of geometry of the analyzed area, while the FEM formulation can be succesfully used for domains of high complexity. The developed computer program is robust and flexible, and it can be easily modified to solve different equations or to model various physical phenomena.

\section{References}

[1] Tichonow, A.N., \& Samarski, A.A. (1963). Równania fizyki matematycznej. Warszawa: PWN.

[2] Zaporożec, G. (1967). Metody rozwiazywania zadań z analizy matematycznej. Warszawa: WNT.

[3] Fichtenholz, G.M. (1999). Rachunek różniczkowy i całkowy. Warszawa: Wyd. Nauk. PWN.

[4] Cichoń, C. (2005). Metody obliczeniowe. Wybrane zagadnienia. Kielce: Wydawnictwo Politechniki Świętokrzyskiej.

[5] Majchrzak, E., \& Mochnacki, B. (2004). Numerical Methods. Theory, Numerical Aspects, Algorithms. Gliwice: Publication of the Silesian University of Technology.

[6] Taylor, R.L., Zienkiewicz, O.C., \& Zhu, J.Z. (2005). The Finite Element Method: Its Basis and Fundamentals, 6 edition. Oxford: Butterworth-Heinemann.

[7] Pozorska, J., Pozorski, Z., \& Janik, Ł. (2017). Numerical simulations of structural behavior of sandwich panels subjected to concentrated static loads. Journal of Applied Mathematics and Computational Mechanics, 16(2), 113-121.

[8] Suliga, M. (2014). Analysis of the heating of steel wires during high speed multipass drawing process. Archives of Metallurgy and Materials, 59(4), 1475-1480.

[9] Węgrzyn-Skrzypczak, E., \& Skrzypczak, T. (2017). Analytical and numerical solution of the heat conduction problem in the rod. Journal of Applied Mathematics and Computational Mechanics, 16(4), 79-86.

[10] http://math.uni.lodz.pl/ karpinw/zadania/RRCz1/Laplacea_zadania.PDF 